Pfizer, Roche, UCB, Lilly, M. de Hooge Employee of: Selfemployed without other personnel (registered company under the Belgium law) MdH Research. Additional Affiliation: Ghent University, Ghent, Belgium., R. Lambert Consultant for: AbbVie, Bioclinica, Janssen, Parexel, UCB, R. Landewé Grant/research support from: Abbott, Amgen, Centocor, Novartis, Pfizer, Roche, Schering-Plough, UCB, Consultant for: AbbVie, Ablynx, Amgen, Astra-Zeneca, Bristol Myers Squibb, Celgene, Janssen, Galapagos, GlaxoSmithKline, Novartis, Novo-Nordisk, Merck, Pfizer, Roche, Schering-Plough, TiGenix, UCB, Employee of: Director of Rheumatology Consultancy BV, which is a registered company under Dutch law, Speakers bureau: Abbott/AbbVie, Amgen, Bristol Myers Squibb, Janssen, Merck, Pfizer, Roche, Schering-Plough, UCB, A. Molto Grant/research support from: Pfizer, Consultant for: Merck,UCB, D. van der Heijde Consultant for: AbbVie, Amgen, Astellas, AstraZeneca, BMS, Boehringer Ingelheim, Celgene, Daiichi, Lilly, Galapagos, Gilead, GlaxoSmithKline, Janssen, Merck, Novartis, Pfizer, Regeneron, Roche, Sanofi, Takeda, UCB, Employee of: Director of Imaging Rheumatology bv., J. Bukowski Shareholder of: Pfizer, Employee of: Pfizer, H. Jones Shareholder of: Pfizer, Employee of: Pfizer, I. Logeart Shareholder of: Pfizer, Employee of: Pfizer, L. Marshall Shareholder of: Pfizer, Employee of: Pfizer, R. Pedersen Shareholder of: Pfizer, Employee of: Pfizer, A. Szumski Employee of: InVentiv Health, B. Vlahos Shareholder of: Pfizer, Employee of: Pfizer, M. Dougados Grant/research support from: Pfizer, AbbVie, UCB, Merck, Lilly, Consultant for: Pfizer, AbbVie, UCB, Merck, Lilly DOI: 10.1136/annrheumdis-2018-eular.1114

THURSDAY, 14 JUNE 2018

\section{Reproductive issues in rheumatology}

\section{OP0200 CHARACTERISTICS AND OUTCOMES OF PROSPECTIVELY REPORTED PREGNANCIES EXPOSED TO CERTOLIZUMAB PEGOL FROM A SAFETY DATABASE}

M.E.B. Clowse ${ }^{1}$, A.E. Scheuerle ${ }^{2}$, C. Chambers ${ }^{3}$, A. Afzali ${ }^{4}$, A. Kimball ${ }^{5}$, J. J. Cush ${ }^{6}$, M. Cooney ${ }^{7}$, L. Shaughnessy ${ }^{7}$, M. Vanderkelen ${ }^{8}$, F. Förger ${ }^{9}{ }^{1}$ Duke University Medical Center, Durham, NC; ${ }^{2}$ UT Southwestern Medical Center, Dallas, $T X ;{ }^{3}$ UC San Diego School of Medicine, La Jolla, CA; ${ }^{4}$ Ohio State University, Wexner Medical Center, Columbus, $\mathrm{OH} ;{ }^{5}$ Beth Israel Deaconess Medical Center and Harvard Medical School, Boston, MA; ${ }^{6}$ Baylor Scott and White Research Institute, Dallas, TX; ${ }^{7}$ UCB Pharma, Raleigh, NC, USA; ${ }^{8}$ UCB Pharma, Brainel'Alleud, Belgium; ${ }^{9}$ Inselspital, University of Bern, Bern, Switzerland

Background: Anti-tumour necrosis factor medications (anti-TNFs) are effective in controlling chronic inflammatory diseases, but information about their use and safety in pregnancy is limited. Consequently, anti-TNFs are often discontinued early in gestation. Certolizumab pegol (CZP), an Fc-free, PEGylated anti-TNF approved for treatment of rheumatic diseases and/or Crohn's disease, has no active placental transfer.

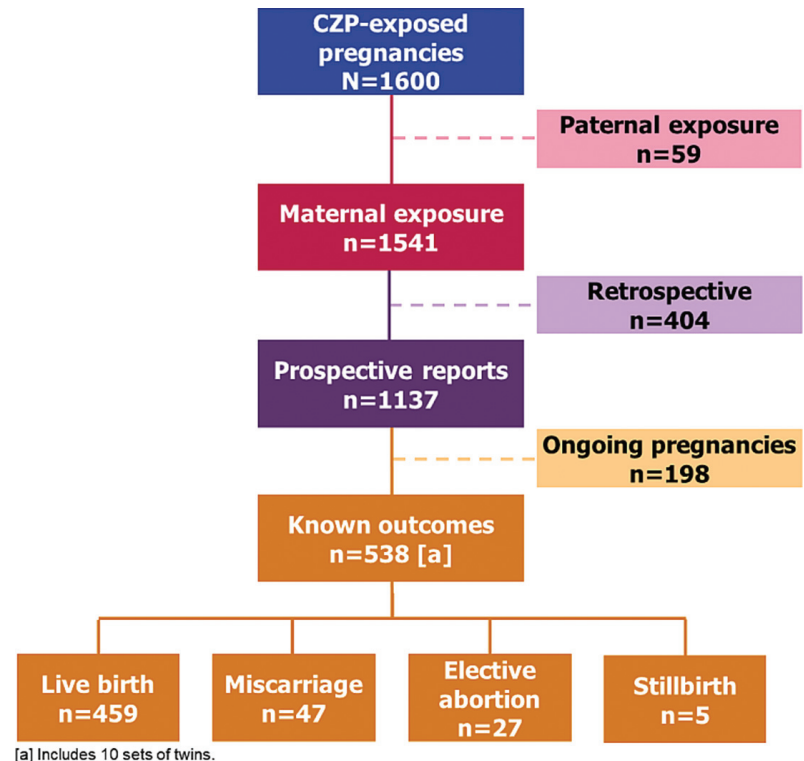

Abstract OP0200 - Figure 1 Overview of pregnancy reports
Objectives: This project provides information on pregnancy outcomes in women receiving CZP, especially those with early pregnancy exposure.

Methods: Prospective and retrospective data on maternal CZP exposure, includ ing timing and duration, outcomes, comorbidities, and major malformations were extracted from the UCB Pharma safety database through 6 March 2017. This analysis was limited to prospective reports to avoid bias associated with retrospective submissions. Numbers of live births, miscarriages, elective abortions stillbirths, and major congenital malformations were ascertained.

Results: From a total of 1541 maternal CZP-exposed pregnancies, 1137 were reported prospectively, of which 528 pregnancies (including 10 twin pregnancies) had 538 known outcomes: 459 live births (85\%), 47 miscarriages (9\%), 27 elective abortions (5\%), and 5 stillbirths (1\%) (figure 1). Of the 459 live births, $8(2 \%)$ cases of major congenital malformations were reported (vesicoureteral reflux, club foot, congenital heart disease, cerebral ventricle dilatation, polydactyly, anal fistula accessory auricle, and hydronephrosis). Of the 528 prospective pregnancies with known outcomes, 436 (83\%) were exposed during the 1 st trimester, when most organogenesis occurs; 201 pregnancies were exposed during the entire pregnancy.

Conclusions: This analysis represents the largest published cohort of pregnant women exposed to an anti-TNF for management of chronic inflammatory diseases. Analysis of pregnancy outcomes does not indicate a malformative effect of CZP compared to the EU general population ( $2 \%-3 \%$ ), nor an increased risk of foetal death. These data are reassuring for women of childbearing age considering treatment with CZP; however, the ongoing collection of post-marketing surveillance data, including the ongoing MotherToBaby study from the Organisation of Teratology Information Specialists, will provide further important information.

Acknowledgements: This study was funded by UCB Pharma. The authors thank the patients and their caregivers in addition to the investigators and their teams who contributed to this study. Editorial services were provided by Costello Medical. All costs associated with development of this abstract were funded by UCB Pharma.

Disclosure of Interest: $M$. Clowse Grant/research support from: Pfizer and Janssen, Consultant for: UCB Pharma, A. Scheuerle Grant/research support from: UCB Pharma, INC Research and Genentech, C. Chambers Grant/research support from: AbbVie, Amgen Inc., Bristol-Myers Squibb, Celgene, GlaxoSmithKline, Janssen, Pfizer, Hoffman-La Roche, Genentech, Genzyme Sanofi-Aventis, Seqirus, Takeda and UCB Pharma, A. Afzali Grant/research support from: UCB Pharma, Consultant for: UCB Pharma, AbbVie, Takeda and IBD Horizons, A. Kimball Consultant for: UCB Pharma, Dermira, Janssen and AbbVie, J. Cush Grant/ research support from: Pfizer, Janssen, AbbVie, Celgene, Novartis, AstraZeneca and Genentech, Consultant for: Janssen, AbbVie, Novartis, Amgen, Genentech, Lilly and Horizon, M. Cooney Employee of: UCB Pharma, L. Shaughnessy Employee of: UCB Pharma, M. Vanderkelen Employee of: UCB Pharma, F. Förger Grant/research support from: UCB Pharma, Speakers bureau: Mepha, Roche and UCB Pharma

DOI: 10.1136/annrheumdis-2018-eular.2417

THURSDAY, 14 JUNE 2018

Do we still need biopsies to diagnose Sjögren's and autoimmune myostitis?

\section{OP0201 CLINICAL OUTCOMES AND RESPONSE TO ANTI- THROMBOTIC TREATMENT AMONG PATIENTS WITH CONCOMITANT LUPUS NEPHRITIS AND THROMBOTIC MICROANGIOPATHY: A MULTICENTER COHORT STUDY}

S. Sciascia ${ }^{1}$, J. Yazdany ${ }^{2}$, M. Cuadrado ${ }^{3}$, M. Radin ${ }^{4}$, M. Dall'Era ${ }^{5}$, I. Aggarwal $^{2}$, R. Fenoglio ${ }^{1}$, A. Barreca ${ }^{6}$, M. Papotti ${ }^{6}$, I. Cecchi ${ }^{1}$, E. Rubini ${ }^{1}$, K. Schreiber ${ }^{3}$, D. Roccatello ${ }^{1} .{ }^{1}$ Center of Immunopathology and Rare Diseases, University of Torino, Torino, Italy, ${ }^{2}$ UCSF, San Francisco, USA; ${ }^{3}$ Lupus Unit, GSTT, London, UK; ${ }^{4}$ University of Torino, Torino, Italy; ${ }^{5}$ Center of Immunopathology and Rare Diseases, UCSF, San Francisco, USA; ${ }^{6}$ Pathology, University of Torino, Torino, Italy

Background: In addition to glomerular lesions, renal vascular involvement is an important prognostic marker of lupus nephritis (LN). Among patients with various vascular changes, individuals with thromboticmicroangiopathy (TMA) present with severe clinical manifestations and have a high mortality.

Objectives: We sought to assess renalout comes and response to anti-thrombotic treatmentsin addition to conventional immunosuppression in patients with biopsy proven LN and TMA.

Methods: Clinical and renal histopathological data for 97 patients with biopsyproven LN and TMA were retrospectively analysed. Antibody profiles, induction and maintenance therapies for LN, and anti-thrombotic treatments were collected. TMA lesions were classified into acute and chronic (table 1). A complete renal response $(C R)$ was defined as proteinuria $<0.5 \mathrm{~g} / 24$ hour and normal or near 
normal (within $10 \%$ of normal GFR if previously abnormal) GFR. Partial Response (PR) was defined as $a \geq 50 \%$ reduction in proteinuria to subnephrotic levels and normal or near-normal GFR. Renal outcomes were assed at one year post biopsy.

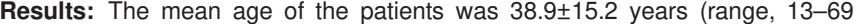
years). The study included 85 females $(87.6 \%)$ and 12 males (12.4\%). The clinical presentations were nephrotic syndrome, nephritic syndrome, and asymptomatic urinary abnormalitiesin 38 (39.2\%), $20(20.6 \%), 39(40.2 \%)$ patients, respectively. Nine patients were classified Class III $(9.3 \%$, including 2 as ClassIII $+\mathrm{V}), 82$ as Class IV (84.5\%, 10 as Class IV-segmental(IV-S) (10.3\%) and 72 as Class IVglobal (IV-G) (74.2\%), including 4 as Class IV-G+V) and 6 as Class V (6.2\%). Forty-two(43\%) patients presented with acute and $55(57 \%)$ with features of chronic TMA. All patients had received treatment with standard immunosuppressants (55\% mycophenolate, 39\% cyclophosphamide, $6 \%$ other regimen) and steroids.

Abstract OP0201 - Table 1

\begin{tabular}{|l|l|}
\hline Acute TMA features & Chronic TMA features \\
\hline -Endothelial swelling with partial or & -Capillary wall thickening with double \\
complete occlusion of lumina; & contours; \\
- Microthrombi, focal or global; & -Organizing capillary thrombi; \\
fragmented RBC on glomerular & -Glomerularischemic collapse with \\
subendothelial space and/or & afferent arteriolar occlusion; \\
mesangial areas; & -Segmental/global glomerulosclerosis \\
-Mesangiolysis, focal, & \\
segmental/global; & \\
-Glomerularcongestion with efferent & \\
arteriolarocclusion & \\
\hline
\end{tabular}

At 12 months, CR was observed in 37 patients (38.1\%), PR in $22(22.6 \%)$ and no response in $38(39.1 \%)$. Sixty-one patients $(62.9 \%)$ were antiphospholipid positive $(\mathrm{aPL})$ and $37(38.1 \%)$ received anticoagulation with vitamin-K antagonist (VKA) and/or heparins. Presence of aPLs(OR, 2.4; 95\% confidence interval-Cl-, $1.2-$ 7.3; $\mathrm{p}=0.03)$, anti-DNA positivity (OR, $12.8 ; 95 \% \mathrm{Cl}: 3.0$ to $71.3 ; \mathrm{p}=0.002)$, and chronic features of TMA (OR, 3.0; $95 \% \mathrm{Cl}: 1.2$ to $17.5 ; \mathrm{p}=0.04)$ were all found to be associated with no response. When limiting the analysis to aPL positive patients, after adjusting for type of immunosuppressant therapy and LN class on biopsy, variables that were significantly associated with $\mathrm{CR}+\mathrm{PR}$ were features of acute TMA rather than chronic (OR, 8.62; 95\% Cl: 1.4 to $97.1 ; \mathrm{p}=0.03)$ and the use of VKA/heparins(OR, 2.1; 95\% Cl, 1.02-16.2; $\mathrm{p}=0.046)$

Conclusions: In patients with concomitant LN and TMA, the presence of aPL and chronic features of TMA were associated with poorer renal outcomes. In patients with $\mathrm{aPL}$, the use of anticoagulation appeared protective and warrants further investigation as a therapeutic tool, especially in the setting of acute TMA. Disclosure of Interest: None declared DOI: 10.1136/annrheumdis-2018-eular.6216

\section{OP0202 GENE EXPRESSION PROFILES IN PRIMARY SJÖGREN'S SYNDROME WITH AND WITHOUT SYSTEMIC MANIFESTATIONS}

C. Vitali ${ }^{1}$, M. Dolcino ${ }^{2}$, R. Andracco ${ }^{3}$, A. Pelosi ${ }^{4}$, P. Fiore ${ }^{4}$, W. Maglione ${ }^{3}$ E. Zaccara ${ }^{3}$, N. Del Papa ${ }^{3}$, A. Puccetti ${ }^{4}$. ${ }^{1}$ Dept. Rheumatology, San Giuseppe Institute, Como; ${ }^{2}$ Dept. of Medicine, University of Verona, Verona; ${ }^{3}$ Dept. Rheumatology, ASST G. Pini-CTO, Milano; ${ }^{4}$ Immunology Area, Bambino Gesù Hospital, Rome, Italy

Background: Different phenotypes characterise the clinical spectrum of primary Sjögren's syndrome (SjS). Patients with a clinical expression limited to glandular features (GFs) are classically distinguished from patients with extra-glandular manifestations (EGMs). The former patients often complain higher level of fatigue and widespread pain (WP). (Segal et al. 2013) This suggests that gene expression pattern may be different in the two subgroups.
Objectives: To investigate the differences of gene expression in SjS patients with GFs and in those with EGMs.

Methods: Nineteen patients with SjS were selected for the study. Gene expres sion in peripheral blood mononuclear cells (PBMCs) was analysed in 4 patients with EGMs and 4 patients with GFs alone using Clariom D human Affymetrix gene chip (Affymetrix, Santa Clara, CA, USA), and compared to that found in healthy controls. Differences in gene expression were evaluated by analysis of variance (ANOVA) and Step-Up FDR-controlling procedure, being FDR corrected $p$ value $\leq 0.01$ and fold change $>2$ considered as statistically significant.

Validation of the gene overexpression was performed by quantitative Real Time (qRT)-PCR in PBMCs from all the selected SjS patients, using the $\Delta \Delta \mathrm{Ct}$ method for comparing relative fold expression differences.

Results: All the enrolled SjS patients (18 females and 1 male) had a positive lip biopsy, while anti-SSA/Ro antibodies were detected in 10/11 and 6/8 of the patients with EGMs and with GFs alone, respectively. ESSDAI value ranged from 7 to 55 in patients with EGMs (median 17), and from 0 to 2 in patients with GFs alone (median 1).

In both types of patients, the functional analysis of the two transcriptomes showed a large number $(>1000)$ of modulated genes that are involved in the well-known pathological processes of SjS, i.e., apoptosis, inflammatory response, immune response, type I and type II interferons, and Toll-like receptors signalling.

Genes modulated only in patient with EGMs showed a significant enrichment of the biological processes associated with immune response $(79 \%$ of all enriched processes) and, namely, of the molecular pathways related to B cell activation. The analysis of the transcripts expressed only in patients with GFs alone showed instead a preponderant enrichment in different metabolic processes (43\%) and in processes associated with the central perception of the stimuli. Indeed, genes involved in sensory perception and in nociceptive signals (i.e., ANPEP, TNRF1 P2RY1, IFNG) were modulated exclusively in patients with GFs alone. The significant differential expression of selected genes in the two SjS subgroups was confirmed by the qRT-PCR analysis.

Conclusions: These data indicate that in SjS patients with GFs alone a dysregu lation of pain pathways (namely beta-adrenergic receptor and Notch signalling may play a role in the development of WP that is common in this subset of patients. The biological mechanisms triggering the activation of these genes remain to be completely clarified.

Disclosure of Interest: None declared

DOI: 10.1136/annrheumdis-2018-eular.5119

\section{THURSDAY, 14 JUNE 2018}

\section{How monogenetic autoinflammatory diseases help to understand and treat rheumatic diseases}

\begin{tabular}{|l|l}
\hline OP0203 & SAFETY AND EFFICACY OF INTRAVENOUS \\
& ADMINISTRATION OF BONE-MARROW DERIVED \\
& MESENCHYMAL STROMAL CELLS IN THERAPY \\
& REFRACTORY JUVENILE IDIOPATHIC ARTHRITIS \\
& PATIENTS, A PHASE IB/IIA PILOT-STUDY
\end{tabular}

J.F. Swart ${ }^{1}$, S. de Roock ${ }^{1}$, R.J. Nievelstein ${ }^{2}$, I. Slaper-Cortenbach ${ }^{3}$, J.J. Boelens ${ }^{4}$ N. Wulffraat ${ }^{1} .{ }^{1}$ Pediatric Rheumatology; ${ }^{2}$ Pediatric Radiology; ${ }^{3}$ Cell Tharpy Facility; ${ }^{4}$ Pediatric Immunology, UMC UTRECHT, Utrecht, Netherlands

Objectives: To compare the total number of adverse events (AE's) before and after mesenchymal stromal cell (MSC) infusion in refractory JIA and to evaluate its effectiveness.

Methods: Single-centre Phase lb/lla, open label intervention study in JIA patients previously failing all biologicals registered for their diagnosis. Six patients will receive 2 million $/ \mathrm{kg}$ intravenous infusions of allogeneic bone-marrow derived MSC. In case of ACR-Ped30-response but subsequent loss of response one and maximal two repeated infusions are allowed.

Results: Six JIA patients with 9.2 years median disease duration, still active arthritis and damage were included. All had failed methotrexate, corticosteroids and median 5 different biologicals. MSC were administered twice in 3 patients. No acute infusion reactions were observed and a lower post-treatment than pre-treatment incidence in AE's was found. The one sJIA patient had again an evolving macrophage activation syndrome, 9 weeks after tocilizumab discontinuation and 7 weeks post- MSC infusion.

Eight weeks after one MSC infusion, 4 patients showed less active joints, 5 patients improved in many clinical parameters and inflammatory parameters decreased in $3 / 4$. After 1 year, we found significantly lower active joint counts, 